\title{
Representation of the evolution of the Universe in the JBD theory considering vacuum phenomena
}

\section{G.H. Harutyunyan*}

Department of Physics, Yerevan State University, Alex Manoogian 1, Yerevan, 0025, Armenia E-mail: hagohareysu.am

\section{R.M. Avagyan}

Department of Physics, Yerevan State University, Alex Manoogian 1, Yerevan, 0025, Armenia

\section{A.S. Kotanjyan}

Department of Physics, Yerevan State University, Alex Manoogian 1, Yerevan, 0025, Armenia

\section{A.V. Hovsepyan}

Department of Physics, Yerevan State University, Alex Manoogian 1, Yerevan, 0025, Armenia

\begin{abstract}
One of the currently available approaches to the problem of the accelerated expansion of the Universe is based on cosmological models with various types of scalar fields. In this paper we consider a cosmological model based on the modified Jordan-Brans-Dicke (JBD) theory with a cosmological scalar $\varphi(y)$ playing the role of the variable cosmological constant. Special cases are considered with $\varphi(y)=\alpha H^{2}$ and $\varphi(y)=\alpha H^{4}$. In particular, we show the possibility of the expansion with uniform acceleration.
\end{abstract}

The Modern Physics of Compact Stars 2015

30 September 2015 - 3 October 2015

Yerevan, Armenia

\footnotetext{
${ }^{*}$ Speaker.
} 


\section{Introduction}

In a series of papers [1]-[3] we have constructed different cosmological models of the de Sitter type on the basis of the modified JBD scalar-tensor theory of gravity, by taking into account the interaction of the vacuum and scalar fields, which in addition to the kinetic energy is described by a cosmological scalar $\varphi(y)$. The latter is introduced in analogy with the cosmological constant $\Lambda$. Essentially, the combination $y \varphi(y) / k^{2}$ plays the role of the potential energy for the above mentioned scalar field. As a result, in all the problems considered the expansion of the Universe at late stages is qualitatively similar. The expansion is accelerating and the time-dependence of the scale factor is not exponential. In all cases, the parameter $q$ ("deceleration parameter") tends to 1 . The qualitative picture of the time-dependence of the energy contributions for the scalar field, $\Omega_{s c}$, and $\Omega_{\Lambda}$ in all the cases is the same.

By taking into account known considerations [4] on the existence of the relationship between $\Lambda$ and the Hubble parameter $H$, in the first section of the paper we assume that $\varphi(y)=\alpha H^{2}$. This assumption can also be argued on the base of the time-dependence of $H$ given in [5], where it has been shown that in the limit $t \rightarrow \infty$ one has $H^{2}=\Lambda / 3$. We consider a cosmological model in the absence of the ordinary matter. As a result, a uniformly accelerated expansion is obtained with the scale factor different from that discussed in the papers mentioned above. For the maximal possible value of $\alpha_{\max }$ the model becomes de Sitter one. Consequently, we also consider the de Sitter model with the equation of state $P=-\varepsilon$.

In the second part of the paper, within the framework of two different conformal representations of the modified JBD theory, inflationary regimes are constructed with the specific potential of the scalar field $\varphi(y)=\alpha H^{4}$.

\section{Uniformly accelerated expansion of the Universe}

Here we consider the de Sitter cosmological model in the proper presentation of the JBD theory with $P=0, \varepsilon=0, \varphi(y)=\alpha H^{2}$. The equations for the standard cosmological model, corresponding to the modified action of the JBD theory [4]

$$
W=\frac{1}{c} \int \sqrt{-g}\left[-\frac{y}{2 k}(R+2 \varphi(y))+\zeta g^{\mu v} \frac{y_{\mu} y_{v}}{y^{2}}\right] d^{4} x,
$$

in the notations $\psi=\dot{y} / y, H=\dot{a} / a$, have the form

$$
\begin{gathered}
\dot{\psi}+\psi^{2}+3 \psi H=\frac{2 \alpha H^{2}}{3+2 \zeta}\left(1-\frac{2 \dot{H}}{H \psi}\right), \\
2 \dot{H}+3 H^{2}=-\dot{\psi}-\psi^{2}(1+\zeta / 2)-2 \psi H+\alpha H^{2}, \\
3 H^{2}=\frac{\zeta \psi^{2}}{2}-3 \psi H+\alpha H^{2} .
\end{gathered}
$$

Here, $\zeta$ is the coupling constant between the metric and the scalar field. The cosmological scalar $\varphi(y)=\alpha H^{2}$ ( $\alpha$ being a dimensionless constant) plays the role of the vacuum energy density. This choice of the cosmological scalar leads to the relation

$$
\frac{\psi}{H}=\frac{3 \pm \sqrt{9+2 \zeta(3-\alpha)}}{\zeta}=\gamma
$$


with the restriction $\alpha \leq 3+9 /(2 \zeta)$. Taking into account (2.5), from (2.2), (2.3) one can get

$$
\frac{\dot{H}}{H^{2}}=\gamma \frac{1-\gamma(1+\zeta)}{2+\gamma}=-\sigma
$$

From here we obtain the time dependence of the Hubble parameter $H$, of the scale factor $a$ and of the scalar potential $y$ :

$$
\begin{gathered}
\frac{H}{H_{0}}=\frac{1}{1+\sigma t^{\prime}}, \\
\frac{a}{a_{0}}=\left(1+\sigma t^{\prime}\right)^{1 / \sigma}, \\
\frac{y}{y_{0}}=\left(\frac{a}{a_{0}}\right)^{\gamma}=\left(1+\sigma t^{\prime}\right)^{\gamma / \sigma},
\end{gathered}
$$

with

$$
t^{\prime}=H_{0}\left(t-t_{0}\right), \quad q=1-\sigma .
$$

Here $H_{0}, a_{0}, y_{0}$ are the values of the corresponding functions at recent time $t_{0}$, and $q=\ddot{a} a / \dot{a}^{2}$ is the dimensionless "deceleration" parameter. It is of interest to consider these relations for the limiting value $\alpha_{\max }=3+9 /(2 \zeta)$, for which one has $\gamma=\sigma=3 / \zeta$. The scale factor evolves in accordance with

$$
a=a_{0}\left(1+\frac{3}{\zeta} t^{\prime}\right)^{\zeta / 3}
$$

In the Einsteinian limit $\zeta \rightarrow \infty$, by taking into account the formula $\lim _{n \rightarrow \infty}(1+x / n)^{n}=e^{x}$, one gets an exponential expansion $a=a_{0} e^{H_{0}\left(t-t_{0}\right)}$, which corresponds to the uniformly accelerated evolutionary scenario with $q=1$. Note that

$$
\frac{\dot{\psi}}{H^{2}}=\frac{3}{\zeta} \frac{\dot{H}}{H^{2}}=-\frac{9}{\zeta^{2}} \rightarrow 0,
$$

and, hence, $H=H_{0}$ and $\psi=\psi_{0}$. For the equation of state one has $P=-\rho \approx-3 H^{2}$. Thus, for $\alpha=\alpha_{\max }$, in the absence of the ordinary matter, the model of the uniformly accelerated expansion of the Universe is of the de Sitter type within the framework of general relativity in the presence of the cosmological scalar $\varphi(y)=\alpha H^{2}$. Therefore, it makes sense to consider the problem of de Sitter cosmological model with the equation of state $P=-\varepsilon$ and with the cosmological scalar $\tilde{\varphi}(y)=\tilde{\alpha} H^{2}$ for arbitrary values of $\zeta$. Denoting by $\Omega_{0}=8 \pi G \varepsilon_{0} /\left(3 H^{2} y\right)$ the contribution of the vacuum energy, the analogs of equations (2.2)-(2.4) are written as

$$
\begin{gathered}
\frac{\dot{\psi}}{H^{2}}+\tilde{\gamma}^{2}+3 \tilde{\gamma}=\frac{2}{3+2 \zeta}\left(6 \Omega_{0}+\tilde{\alpha}-\frac{2 \tilde{\alpha} \dot{H}}{\tilde{\gamma} H^{2}}\right), \\
\frac{2 \dot{H}}{H^{2}}=-\frac{\dot{\psi}}{H^{2}}-\tilde{\gamma}^{2}\left(1+\frac{\zeta}{2}\right)-2 \tilde{\gamma}^{2}+\tilde{\alpha}-3+\frac{3 \Omega_{0}}{3+2 \zeta}, \\
1=\Omega_{0}+\frac{\zeta \tilde{\gamma}^{2}}{6}-\tilde{\gamma}+\frac{\tilde{\alpha}}{3} .
\end{gathered}
$$

Excluding from the equations (2.13) and (2.14) $\dot{\psi}$, one can obtain

$$
\frac{\dot{H}}{H^{2}}=q-1=\frac{1}{2} \frac{(1+2 \zeta) \zeta \tilde{\gamma}^{2}-8 \zeta \tilde{\gamma}-2 \alpha+12}{2 \alpha / \tilde{\gamma}-3-2 \zeta} \equiv-\tilde{\sigma}
$$


From (2.15) we have

$$
\tilde{\gamma} \equiv \frac{\psi}{H}=\frac{3 \pm \sqrt{9-2 \zeta\left(\alpha-3+3 \Omega_{0}\right)}}{\zeta}
$$

with the constraint $\tilde{\alpha} \leq 3-3 \Omega_{0}+9 / 2 \zeta$.

For given $\tilde{\gamma}$ and $\tilde{\sigma}$, the results of the integration have the same form as (2.7)-(2.10). Again we get $q=1-3 / \zeta$. The difference from the first variant is the presence in the model of the vacuum energy density with $\Omega_{0} \approx 0.7$. The behavior of the Hubble function, scale factor and the scalar field is shown in figures 2 and 3 for $\alpha=2.5$.

In Figure 1 we have presented the parameter $q$ as a function of $\alpha$. It is remarkable that for the maximum allowed value $\alpha_{\max }=3+9 /(2 \zeta)$, for large $\zeta$ this version of theory is equivalent to the de Sitter model in the framework of the Einstein theory in the presence of the cosmological scalar $\varphi(y)=\alpha H^{2}$. In the left panel of Figure 2 we displayed the characteristic time dependence for $H / H_{0}$. The right panel shows the quasi-exponential growth of the scale factor $a$, which is transformed into a purely exponential in the limiting case $\alpha=3+9 /(2 \zeta)$. Figure 3 shows the time dependence of the gravitational scalar $y$ which can be interpreted as gravitational constant $G$, decreasing in time.



Figure 1: The dependence of $q$ on the parameter $\alpha$.

\section{Inflationary models in the "Einstein" representation}

In this section we consider a model with a minimally coupled scalar field in the presence of the cosmological scalar $\varphi(\Phi)$, described by the action

$$
W=\int \sqrt{-g}\left[-\frac{y_{0}}{16 \pi}(R+2 \varphi(\Phi))+\frac{1}{2} g^{\alpha \beta} \Phi_{\alpha} \Phi_{\beta}\right] d^{4} x .
$$

Introducing the notation $y_{0} \varphi(\Phi) /(8 \pi) \equiv V(\Phi)$, the respective field equations take the form

$$
\begin{gathered}
\ddot{\Phi}+3 H \dot{\Phi}+V^{\prime}(\Phi)=0 \\
3 H^{2}=\frac{8 \pi}{y_{0}}\left(\frac{1}{2} \dot{\Phi}^{2}+V(\Phi)\right)=\frac{8 \pi}{y_{0}} \rho_{e f f},
\end{gathered}
$$



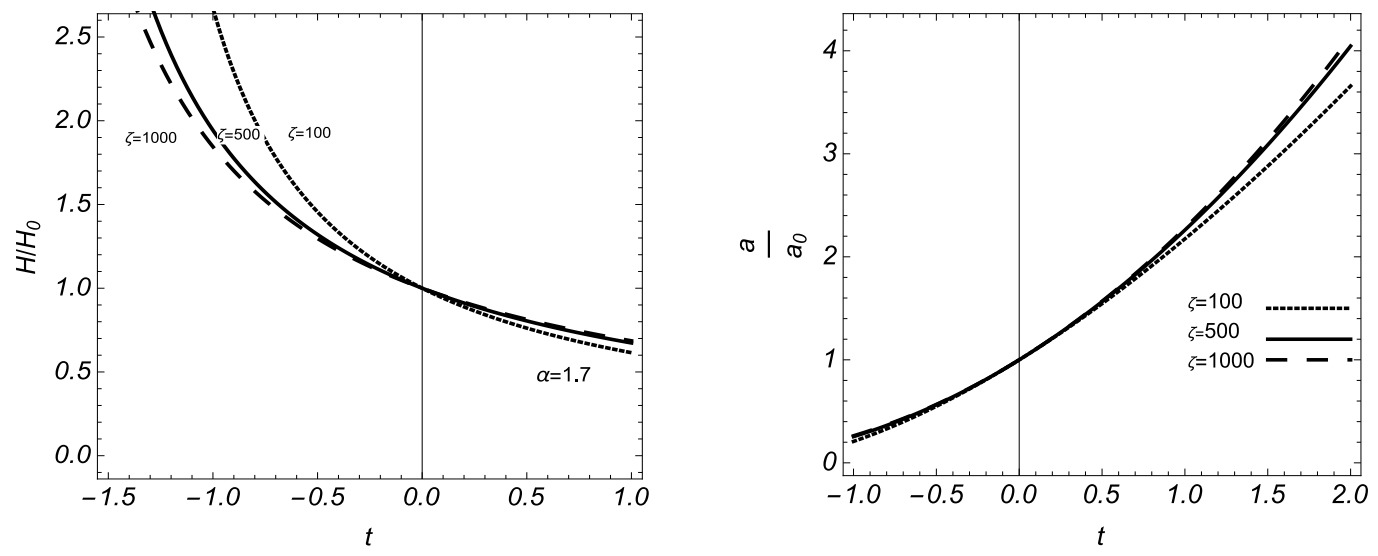

Figure 2: The Hubble function (left panel) and the scale factor (right panel) versus time.

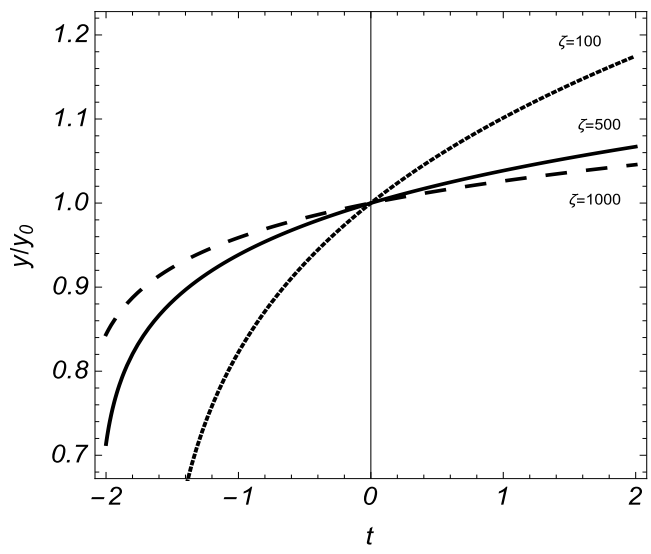

Figure 3: The time dependance of the gravitational scalar $y$.

$$
2 \dot{H}+3 H^{2}=\frac{8 \pi}{y_{0}}\left(-\frac{1}{2} \dot{\Phi}^{2}+V(\Phi)\right)=-\frac{8 \pi}{y_{0}} P_{e f f} .
$$

The corresponding energy-momentum tensor is of the perfect fluid form with the energy density and pressure given by $\rho_{e f f}=\dot{\Phi}^{2} / 2+V(\Phi)$ and $P_{e f f}=\dot{\Phi}^{2} / 2-V(\Phi)$. Here and in what follows the dot stands for the time derivative and the prime means the derivative with respect to $\Phi$.

The form of (3.2) allows us to use a simple physical interpretation: the mechanical rolling in the potential $V(\Phi)$ in the presence of the time-dependent friction coefficient $3 H$ (Hubble friction) [6]-[8].

Inflation in the slow roll-approximation occurs when the term $3 H \dot{\Phi}$, responsible for the friction, dominates the acceleration term $\ddot{\Phi}$ :

$$
\left|\frac{\ddot{\Phi}}{3 H \dot{\Phi}}\right|<<1 .
$$

The Hubble friction leads to the displacement of $\Phi$ in the direction of decreasing of the potential. The second condition for the slow roll-approximation corresponds to the smallness of the kinetic 
term $\dot{\Phi}^{2} / 2$ with respect to the potential one:

$$
\frac{\dot{\Phi}^{2}}{2 V(\Phi)} \ll 1
$$

which leads to the equation of state $P_{e f f} \approx-\rho_{e f f}$ and corresponds to a vacuum type source required for the inflation. Under the conditions (3.5), (3.6), the set of equations takes the form

$$
\begin{gathered}
3 H \dot{\Phi} \approx-V^{\prime}, \\
3 H^{2} \approx V, \\
2 \dot{H}+3 H^{2}=V .
\end{gathered}
$$

From the first two equations we get

$$
2 H^{\prime}=-\dot{\Phi} .
$$

The equations (3.8), (3.9) can be considered as the third condition for the slow roll-approximation

$$
\left|\frac{\dot{H}}{H^{2}}\right| \ll 1 .
$$

We assume that $V=\alpha H^{4}$, take into account Eq.(3.10), and present Eq. (3.8) in the form

$$
4 H^{2}=3 H^{2}-V
$$

in order to determine the dependence of $H(\Phi)$ and $V(\Phi)$. We will consider the case $\alpha>0$.

Introducing the notation

$$
x \equiv \sqrt{\frac{3}{\alpha}} \frac{1}{H},
$$

in the general case, under the condition $\alpha<3 / H^{2}$, the solution of (3.12) can be written as

$$
x=\cosh \left(C+\frac{\sqrt{3}}{2}\left(\Phi_{0}-\Phi\right)\right) \text {. }
$$

Here

$$
C=\operatorname{Arccosh} x_{0}, x_{0} \equiv \sqrt{\frac{3}{\alpha}} \frac{1}{H_{0}}
$$

$H_{0}, \Phi_{0}$ are the initial values of the corresponding quantities. Choosing the constants in accordance with

$$
\Phi_{0}=0, \quad \alpha=3 / H_{0}^{2} \Rightarrow C=0
$$

we get

$$
x \equiv \sqrt{\frac{3}{\alpha}} \frac{1}{H}=\cosh \left(\frac{\sqrt{3}}{2} \Phi\right)
$$

and

$$
\begin{aligned}
\frac{H}{H_{0}} & =\frac{1}{\cosh (\sqrt{3} \Phi / 2)}, \\
V(\Phi) & =\frac{3 H_{0}^{2}}{\cosh ^{4}(\sqrt{3} \Phi / 2)} .
\end{aligned}
$$


The time dependence of $H(t)$ is determined from Eq. (3.9):

$$
2 \dot{H}+3 H^{2}-\frac{3}{H_{0}^{2}} H^{4}=0 .
$$

As a result, the solution can be written as

$$
\frac{3}{2} H_{0} t+\text { const }=\frac{H_{0}}{H}+\frac{1}{2} \ln \left|\frac{H_{0}-H}{H_{0}+H}\right| .
$$

The scalar potential $V(\Phi)$ and the time dependence of the Hubble function are presented in the left and right panels of Figure 4, respectively.
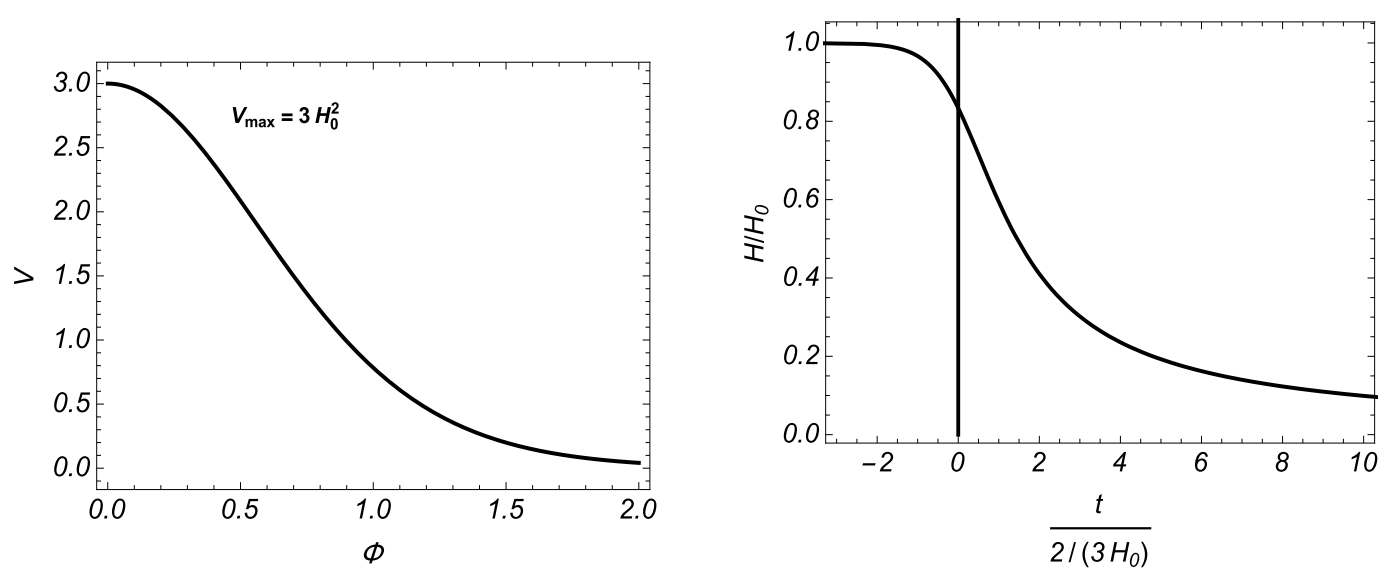

Figure 4: The left panel presents the potential $V(\Phi)$. In the right panel the time dependence of $H / H_{0}$ is plotted.

Another possible inflationary model within the framework of the proper representation of the modified JBD theory can be constructed on the basis of the solution of the problem presented in the previous section for the value $\alpha=\alpha_{\max }$. In fact, this case is realized within the framework of the Einstein theory in the presence of the cosmological scalar $\varphi(y)=\alpha H^{2}$.

\section{Conclusion}

In the present paper, on the basis of the modified JBD theory and under the assumption $\varphi(y) \sim$ $H^{n}$ ( $n$ is a parameter equal to four in the regime of inflation and two - at late stages), we have considered the inflation and late stages of the Universe expansion. For the late stage, a dynamical picture of the uniformly accelerated expansion is obtained with $q=$ const.

\section{Acknowledgments}

The work was supported by the State Committee of Science Ministry of Education and Science RA, within the frame of Research Projects No. SCS 15T-1C110 and No. 15 RF-009. 


\section{References}

[1] R.M. Avagyan, G.H. Harutyunyan, A.V. Hovsepyan, A.S. Kotanjyan, Astrophysics, 56, 471 (2013).

[2] R.M. Avagyan, G.H. Harutyunyan, A.V. Hovsepyan, A.S. Kotanjyan, Astrophysics, 56, 617 (2013).

[3] R.M. Avagyan, G.H. Harutyunyan, A.V. Hovsepyan, A.S. Kotanjyan, Astrophysics, 57, 327 (2014).

[4] R.M. Avagyan, G.H. Harutyunyan, Astrophysics, 53, 317 (2010).

[5] R.M. Avagyan, G.H. Harutyunyan, Astrophysics, 48, 633 (2010).

[6] A.D. Dolgov, Ya.B. Zeldovich, M.V. Sazhin, The cosmology of late Universe, MSU, 1988.

[7] E.T. Copeland, M. Sami, S. Tsujikava, Mod. Phys. D 15, 1753 (2006).

[8] V. Sahni, A.A. Starobinsky, Int. J. Mod. Phys D 9, 373 (2000). 